\section{$\underset{\substack{\text { hommes } \\ \text { \& migrations }}}{ }$}

\section{Hommes \& migrations}

Revue française de référence sur les dynamiques

migratoires

$1295 \mid 2012$

Algérie - France : une communauté de destin

\title{
La participation politique des immigrés algériens et de leurs descendants en France
}

Entretien avec Vincent Tiberj

\section{Marie Poinsot}

\section{(2) OpenEdition}

Journals

Édition électronique

URL : http://journals.openedition.org/hommesmigrations/1071

DOI : 10.4000/hommesmigrations. 1071

ISSN : 2262-3353

Éditeur

Musée national de l'histoire de l'immigration

Édition imprimée

Date de publication : 1 janvier 2012

Pagination : 78-89

ISSN : 1142-852X

Référence électronique

Marie Poinsot, «La participation politique des immigrés algériens et de leurs descendants en France ", Hommes \& migrations [En ligne], 1295 | 2012, mis en ligne le 01 janvier 2014, consulté le 19 avril 2019. URL : http://journals.openedition.org/hommesmigrations/1071; DOI : 10.4000/

hommesmigrations. 1071 


\section{La participation politique des immigrés algériens et de leurs descendants en France Entretien avec Vincent Tiberj Chercheur au Centre d'études européennes de Sciences Po (CEE)}

Réalisé par Marie Poinsot

H\&M : Quels sont les thèmes sur lesquels vous travaillez habituellement ? Pouvez-vous revenir sur l'enquête RAPFI (Rapport au politique des Français issus de l'immigration) réalisée en 2005, ainsi que sur les travaux suivants, et notamment l'enquête TeO (Trajectoires et origines) ?

Vincent Tiberj : Mon premier champ de recherche porte sur la participation électorale, mais aussi sur la sociologie des valeurs et des préjugés, qui croise celle de l'immigration. De ce fait, j'aborde la question du vote et de l'intégration des minorités des deux côtés, à savoir du côté des Français issus de l'immigration et des immigrés, mais aussi de celui de la société d'accueil. À ce propos, j'observe les pratiques de rejet et d'acceptation des populations immigrées, ainsi que leur évolution dans le temps politique.

Je travaille notamment avec Nonna Mayer et Guy Michelat sur les baromètres de la Commission nationale consultative des droits de l'homme. Je mène également des enquêtes électorales traditionnelles, plus spécifiquement sur le champ de l'immigration, avec mon collègue Sylvain Brouard. Sur ces questions, l'enquête Rapport au politique des Français issus de l'immigration (RAPFI) en 2005, que nous avons reproduite en 2008, était une enquête miroir visant à comparer, à partir des mêmes questions, les résultats portant sur un échantillon de Français d'origines maghrébine, africaine et turque et sur un échantillon représentatif de la population française. Cela nous a permis de mesurer les degrés de ressemblance et de dissemblance entre les deux populations. 
Avec Sylvain Brouard, nous avons également réalisé une enquête sur les habitants des zones urbaines et, enfin, entre 2006 et aujourd'hui, j’ai été membre de l'équipe rassemblée autour de Catherine Borrel et de Patrick Simon sur l'enquête Trajectoires et origines (TeO). Cette enquête Insee/Ined est probablement la plus grande enquête sur l'immigration en France depuis une bonne dizaine d'années. L'échantillon est vaste et concerne à la fois les immigrés et leurs enfants. Il a permis d'étudier suffisamment d'individus issus des principaux courants migratoires et de comparer l'immigration récente avec celle plus ancienne, notamment européenne.

H\&M : Dans la synthèse de l'enquête TeO, vous présentez trois niveaux d'analyse principaux. Premièrement, la participation civique des populations étrangères, notamment à travers leur engagement dans les mouvements associatifs. Deuxièmement, leur participation électorale, qui nous oblige aussi à considérer les modalités d'accès au vote, c'est-à-dire aussi les modes de naturalisation des étrangers, puisqu'il faut être français pour voter. Troisièmement, vous évoquez la participation des citoyens d'origine étrangère en tant qu'élus au niveau local, ainsi que les difficultés d’accès au système politique, comme les partis.

Concentrons-nous plus particulièrement sur l'immigration algérienne. En ce qui concerne la participation civique, vous montrez, par exemple, qu'il y a peu de différences dans l'implication dans la vie associative entre la population algérienne, à statut social et niveau d'éducation similaires, et le reste de la population en France.

V. T. : Dans le cadre de ces enquêtes, nous avons posé la question de l'impact de l'origine : est-ce que le fait d'être d'origine immigrée ou descendant d'immigrés a un impact au niveau politique en général ? Par "politique", nous entendons la participation à des groupes associatifs, l'intérêt que l'on peut porter à la municipalité, l'investissement que l'on peut y prendre, le vote, ainsi que le type de parti que l'on va privilégier.

C'est quelque chose d'important, puisque dans la France républicaine, la tendance est de ne pas forcément prendre en compte l'origine comme étant une variable importante : la sociologie électorale française évoque la religion, la classe sociale, le genre, mais très rarement la question de l'origine. En l'occurrence, on commence à avoir des résultats plus convergents, qui nous font penser que le fait d'être d'origine immigrée, ou descendant d'immigrés, a un impact notable sur la participation électorale. Par l'impact en question, nous entendons qu'il y a des différences selon les courants migratoires. En particulier, les immigrés d'origine algérienne sont dans une situation différente par rapport aux immigrés arrivés en même temps 
qu'eux, comme les Portugais et les Italiens. Cette situation particulière se reproduit également parmi leurs descendants.

D'autre part, il semblerait que, sur la question de l'intérêt politique, le fait d'être immigré ou descendant d'immigrés n'a pas l'impact généralement escompté. À niveau de diplôme égal, les immigrés et les enfants d'immigrés s'avèrent plus intéressés par la politique que les Français sans ascendance étrangère. En effet, quand on réfléchit à la question de l'impact de l'origine, il faut aussi considérer que la société d'accueil a une responsabilité d'animation des effets possibles des origines.

H\&M : Concernant la participation associative, le tableau que vous dressez montre que les immigrés algériens sont adhérents pour $15 \%$ à des associations, alors que les Espagnols, les Italiens et les autres populations d'origine européenne participent visiblement davantage, à hauteur de $33 \%$ et $38 \%$. Ces immigrés algériens n'adhèrent peut-être pas au même type d'associations que les immigrés d'origine européenne. D'autre part, leurs descendants illustrent un renforcement très net de leur participation associative. Ces données permettent de botter en touche l'idée de communautarisme, d'une communautarisation de la vie des immigrés originaires d'Algérie via justement une pratique associative qui viserait à développer l'entre-soi et l'enfermement sur la culture et l’identité.

V. T. : Si l'on reprend les données de l'enquête faite au milieu des années quatrevingt-dix, on constate, par exemple, que les immigrés portugais étaient adhérents à des associations - et cela est encore vrai aujourd'hui - et fréquentaient des associations culturelles portugaises. Or, si l'on regarde de plus près, on se rend compte que les immigrés algériens participent moins à des associations dont les adhérents ont la même origine qu'eux, c'est-à-dire algérienne, où ils sont très minoritaires, entre $10 \%$ et $15 \%$ des membres.

En revanche, l'immigration turque est tout aussi participative que l'immigration algérienne au niveau associatif, mais elle est beaucoup plus centrée sur la communauté, à hauteur de 50 \% des membres. En ce qui concerne l'immigration originaire d'Afrique subsaharienne, cette proportion est de $33 \%$. Ces populations sont donc très participatives au niveau associatif, mais à cela s'ajoute également une dimension communautaire. L'immigration algérienne a beaucoup moins de mal à s'ouvrir à la société française que l'immigration turque, même s'il faut considérer une grande différence dans la maîtrise de la langue entre les deux groupes. Il faut noter que les immigrés turcs sont dans une situation très proche de 
celle des immigrés d'origine maghrébine en termes de cible des préjugés mais, à la différence des Maghrébins, les Turcs n'ont pas été scolarisés et socialisés dans un environnement où la langue française était très présente.

H\&M : Peut-on dire qu'il y a moins d'associations culturelles qui visent à faire la promotion de la culture algérienne en France et peu de renouvellement de ce type d'associations parmi les générations suivantes?

V. T. : Cette remarque est juste, mais il faut considérer aussi qu'il y a d'autres types d'entités qui permettent à la culture de perdurer, dans la relation avec les parents par exemple. Lorsque l'on pose la question de l'intérêt pour la politique du pays d'origine, on constate qu'il y a une différence très importante entre les immigrés et leurs descendants : 31 \% des immigrés algériens disent s'intéresser à la politique de leur pays d'origine, contre $22 \%$ de leurs descendants, soit largement moins.

H\&M : À propos de cet intérêt pour la vie politique du pays d’origine, vous notez qu'il existe un particularisme algérien par rapport aux deux autres populations d’origine maghrébine, les Tunisiens et les Marocains. 
Quelle est cette singularité qui s'estompe lorsqu'on observe la relation des descendants d'immigrés algériens avec la vie politique du pays d'origine de leurs parents?

v. T. : Pour beaucoup, cela tient à des temporalités migratoires différentes. La migration algérienne en France est contemporaine de celle des Portugais, avant de connaître une résurgence au moment des évènements des années quatre-vingt-dix. En revanche, les migrations marocaine et tunisienne sont des migrations plus récentes. Ainsi, les descendants d'immigrés algériens sont dans un rapport plus distendu avec leur pays d'origine que les Marocains et les Tunisiens. Ce qui différencie les migrations intra-européennes et extra-européennes, c'est la distance géographique au pays d'origine. En effet, les migrations espagnole, portugaise et italienne gardent un rapport étroit avec le pays d'origine qui est entretenu par la fréquence des visites au pays.

H\&M : L'histoire de la colonisation n'aurait donc eu aucune incidence sur la relation de ces migrations algériennes avec leur pays d'origine? Cette histoire n'aurait-elle pas modifié les rapports entre la société algérienne et la société française?

V. T. : Il faut faire attention, car ce type de données est très difficile à saisir lorsque l'on réalise une enquête quantitative comme TeO : par exemple, nous n'avons pas pu poser des questions sur les valeurs. En revanche, dans le cadre de l'enquête RAPFI, on a pu voir que la dimension coloniale, la "dette coloniale", était extrêmement prégnante dans l'immigration maghrébine et africaine, avec une particularité propre à l'Algérie. Cette idée selon laquelle la France a une dette envers les anciennes colonies était davantage présente chez les immigrés algériens et leurs descendants. Ce phénomène est important à souligner, notamment dans le processus d'intégration des immigrés, et dans l'attitude que la société d'accueil manifeste à leur endroit.

\section{H\&M : Cette histoire est-elle un élément déterminant dans le compor- tement et la participation politiques des Algériens dans la société fran- çaise?}

V. T. : Oui, cela est un fait marquant, surtout en termes de réaction. Dans le cadre des enquêtes RAPFI et TeO, nous avons pu constater que, lorsque l'origine a un impact dans les comportements politiques, c'est notamment en raison des préjugés subis par les individus. En 2005, on a pu constater que les Français craignaient plus l'immigration de l'Europe de l'Est que celle d'origine maghrébine. Pourtant, les immigrés est-européens se sentent moins discriminés, moins mis à l'écart, par rapport à ce que perçoivent les immigrés maghrébins. 
Cet écart est dû au fait que, dans un cas comme dans l'autre, la dimension de la visibilité intervient de manière prioritaire. On peut certes avoir peur des immigrés polonais, mais lorsque l'on croise un Polonais dans la rue, on ne le remarque pas forcément. En revanche, on peut avoir peut-être un peu moins peur des immigrés maghrébins, notamment d'un point de vue économique, mais leur visibilité active des préjugés anciens. Ces populations sont clairement renvoyées à une altérité, quand bien même certains immigrés maghrébins et leurs descendants sont très bien intégrés dans la société française et ont réussi socialement. Ils sont renvoyés à leurs origines, à la différence d'autres populations immigrées, comme les Polonais.

On mesure également que la guerre d'Algérie a structuré un certain nombre de préjugés qui perdurent sur le très long terme. Cela apparaît si l'on considère que les immigrés d'origine subsaharienne, et les Noirs en général, sont perçus comme une population étant "moins à part" que les Maghrébins. Il y a une véritable différence dans la perception de ces deux minorités visibles : les Français noirs seront ainsi moins discriminés que les Français maghrébins. Ainsi, on mesure bien comment la fracture coloniale est présente dans la perception de l'autre au sein de la société française.

\section{H\&M : L'investissement politique plus important des immigrés en France, notamment algériens, serait-il davantage lié à ce sentiment de discrimi- nation, à cette expérience de visibilité et de rejet, plutôt qu'au statut social qui les situe au sein des classes populaires ?}

V. T. : Repéré dans le cadre de l'enquête RAPFI, cette tendance a pu être reprise et généralisée grâce à l'enquête TeO. Si l'on prend en compte l'ensemble des variables sociales classiques, comme la propriété de son logement, la religion, la classe sociale, le niveau de revenu, etc., on se rend compte que l'origine a un impact au niveau de la pratique politique, et qu'elle n'apparaît que dans un type de situation spécifique, à savoir lorsque l'on appartient à des minorités visibles faisant l'objet de préjugés négatifs. Par ailleurs, l'enquête $\mathrm{TeO}$ montre que, lorsque l'on appartient à une minorité visible, se produit ce que l'on peut appeler un "sur-vote", ou un "surplacement" à gauche.

\section{H\&M : Ainsi, il n'y aurait aucune raison d'affirmer qu'il existe en France un "vote ethnique", qui serait lié à des particularités culturelles des populations d'origine immigrée. Si ces populations surinvestissent dans la politique suivant des orientations spécifiques, c'est en réaction à la façon dont la société française les situe.}

V. T. : Tout à fait, et l'on retrouve quasiment à l'identique cette situation aux États-Unis, notamment auprès des populations immigrées d'Asie du Sud-Est : 
Vietnam, Laos et Cambodge. En ce qui concerne les élus d'origine étrangère, la problématique est similaire à celle des femmes, à savoir leur cantonnement à des tâches spécifiques, lorsqu'ils seraient élus. Pour les femmes, ces domaines sont traditionnellement l'éducation ou les affaires sociales, tandis que les élus que l'on qualifierait "de la diversité" sont cantonnés à l'intégration, à la politique de la ville, au sport. Globalement, les partis et les militants qui choisissent les élus ont la tendance à reproduire une élite sociale de dominants. L'Assemblée nationale française est l'une des plus vieilles et des plus masculines d'Europe, ce qui prouve qu'il existe un véritable problème de représentation miroir en termes de génération, de genre, d'origine sociale, etc.

\section{H\&M : Cet effet miroir dans la participation et la représentation poli- tiques s'accentue-t-il avec les générations suivantes ?}

v. T. : Oui, car plusieurs paramètres entrent en jeu. D'abord, on constate la persistance du débat sur la légitimité de la représentation de la diversité. À titre de comparaison, dans les partis français, même si l'on a fixé des seuils obligatoires concernant la présence des femmes, la situation réelle est bien plus complexe. Ensuite, même quand les partis respectent ces obligations et intègrent des femmes, il s'agit de femmes qui ressemblent aux hommes en termes de position sociale et d'âge, ce ne sont pas forcément des femmes jeunes et d'origine ouvrière ou d'origine culturelle différente.

\section{H\&M : À ce propos, l’arrivée sur la scène politique de femmes maghré- bines, de jeunes “beurettes”, n'a-t-elle pas été recherchée comme un emblème de la diversité au détriment de jeunes hommes d’origine maghrébine?}

v. T. : Oui, le fait de valoriser une Rama Yade ou une Najat Belkacem est un choix instrumental, qui permet de mettre en avant une femme, jeune et d'origine immigrée, et de satisfaire ainsi trois critères. Cela dit, il faut rendre justice à la participation plus active des femmes d'origine algérienne, comme le montre notre rapport. Cela contredit l'image d'une femme algérienne soumise, parfois isolée, ayant peu de contacts et d'emprise dans la société française. En termes de participation, on constate que les femmes d'origine algérienne votent, elles sont actives, et leurs filles le sont encore davantage. Il faut rappeler qu'il s'agit d'une immigration ancienne. D'un point de vue sociologique, la convergence des comportements se fait petit à petit, sur le long terme, avec ceux de la société française. Les choses évoluent sans qu'il y ait des politiques explicites, grâce à un effet de masse qui se produit par l'éducation notamment. 
H\&M : II n'y a donc pas besoin de mettre en place une politique d'action positive pour stimuler la participation à la vie politique de ces générations d'origine étrangère ? Vous montrez, par exemple, que les banlieues participent tout autant à ce mouvement que les quartiers centraux. Peut-on dire qu'il n'y a pas de désaffection du politique dans les banlieues, qu'il ne s'agit pas de lieux de relégation tels qu'ils sont peints dans les médias ?

V. T. : Oui, mais l'effet de biais reste extrêmement fort. Lorsque la bibliothèque de Villiers-le-Bel a été saccagée pendant les émeutes de 2005, les médias se sont immédiatement intéressés à cet évènement, en négligeant en revanche le fait que, auparavant, cette bibliothèque était très fréquentée, que des jeunes y venaient pour lire. Leur attention s'est portée sur des épiphénomènes en négligeant d'autres réalités. Lorsque j'affirme que l'intégration est en route, il s'agit tout simplement d'observer la progression du niveau culturel et éducatif des descendants d'Algériens sur l'ensemble de la population, et pas simplement de se focaliser sur des comportements ultraminoritaires comme la délinquance, par exemple.

H\&M : II est intéressant de comprendre pourquoi les Français d'origine algérienne et leurs descendants ne souhaitent pas se positionner sur l'échelle gauche-droite, à hauteur de 50 \%, tandis que l'autre moitié affirme pencher très majoritairement pour la gauche. Est-ce le signe d'une perte de confiance de ces populations dans l'élite politique française, ainsi que dans la capacité des partis français de prendre en compte leurs revendications, comme le disent souvent les médias ?

V. T. : Tout d'abord, il faut mesurer et comparer. Si l'on observe la proportion d'immigrés algériens et de leurs descendants qui ne se considèrent ni de gauche, ni de droite, il faut prendre en compte également ce qui se passe pour les Français sans ascendance étrangère. Or on retrouve globalement les mêmes proportions. Ce qui explique le positionnement "ni à gauche, ni à droite" des immigrés algériens et de leurs descendants, ce sont des indicateurs sociologiques classiques (diplôme, âge, profession). Une fois que l'on considère ces variables classiques de la sociologie de la domination, on constate qu'il n'y a pas d'effet spécifique lié à l'origine. Il s'agit d'un point important, car cela signifie que l'on reste dans une logique classique, celle de l'implication dans la politique pour l'ensemble des citoyens français.

L'origine n'est pas une variable de la domination sociale comme les autres. Le fait de ne pas se placer sur l'échiquier politique, pour les Français d'origine immigrée comme pour ceux sans ascendance étrangère, renvoie à l'idée que la politique ne 
joue plus son rôle, à une crise de confiance, à la crise de la représentation politique. Il ne faut pas non plus surestimer le résultat de cette question. Certes, il s'agit d'une question unique dans le cadre d'une enquête publique, mais si on la compare avec des questions posées dans le cadre d'autres enquêtes, comme dans les enquêtes post-électorales du Centre de recherches politiques de Science Po (CEVIPOF) en 2007, où l'on avait réussi à intégrer une question mesurant les origines, 80 \% d'électeurs d'origine maghrébine votent à gauche.

Dans la question TeO, on a décidé de laisser le choix du "non-choix". Lorsque, dans le cas d'une élection, on a à choisir entre la gauche et la droite, les immigrés et descendants d'immigrés sont généralement du côté de la gauche. 80 \% de Français issus de l'immigration favorables à la gauche, ce n'est pas loin des $90 \%$ d'AfroAméricains qui votent pour le parti démocrate depuis le milieu des années soixante, et ce quelle que soit la couleur de la peau du candidat démocrate.

\section{H\&M : Vos recherches analysent ce que vous appelez le "tropisme à gauche". Vous observez un renforcement de ce tropisme d'une géné- ration à l'autre, puisque les immigrés algériens se prononcent à hau- teur de $\mathbf{3 0} \%$ en faveur de la gauche, et les descendants d'Algériens à hauteur de $50 \%$.}

V. T. : Cela est d'autant plus intéressant que cette tendance va à rebours des théories classiques de l'intégration politique : Robert Dahl part du principe selon lequel les immigrés arrivant aux États-Unis ont des spécificités liées à leur communauté mais, à mesure qu'ils s'insèrent dans la société d'accueil, ainsi que leurs enfants, ces spécificités se gomment. Ce qui fait, par exemple, qu'il n'y a pas de vote italoaméricain ou irlando-américain. Ce qui compte pour ces populations, ce ne sont pas les origines mais les déterminants classiques du vote, comme pour l'ensemble des citoyens américains. On retrouve cette situation pour les immigrés d'origine européenne en France. Il y a une convergence, et les logiques de vote des immigrés et des descendants d'immigrés se rapprochent de celles de la population majoritaire. En revanche, cela n'est pas le cas pour les immigrés algériens et leurs descendants ainsi que pour les Turcs, les Afro-Français et les Ultramarins. Dans ces différents cas, on s'aperçoit qu'il y a davantage de tropisme vers la gauche. Plutôt qu'une homogénéisation ou une convergence avec l'ensemble de la société française, il y a une divergence. Le facteur principal est le préjugé dont ils sont la cible : tandis qu'un immigré maghrébin ou africain, tend à ne pas se sentir légitime à revendiquer un traitement différent lorsqu'il considère que l'on n'est pas juste, les descendants de ces immigrés, nés et scolarisés en France, estiment les préjugés auxquels ils sont confrontés non seulement comme intolérables, mais totalement injustes. 
H\&M : Cela va à l'encontre de cette crainte d'un vote musulman quand on évoque le droit de vote des étrangers sur le plan local. Selon vous, cela a-t-il du sens ?

V. T. : L'islam n'est pas un facteur déclenchant du tropisme à gauche, alors que la discrimination en est un. On a ici un exemple parfait de la volonté de contraindre le social dans des logiques d'explications acceptables. La religion est un facteur de positionnement politique dans la sociologie électorale, pas l'origine. Lorsque, en 2005, notre étude a testé l'attachement aux principes et aux politiques de la laïcité des Français d'origine maghrébine, elle a conclu que pas moins de $80 \%$ d'entre eux étaient attachés au principe de la laïcité. On a posé la question des souhaits de scolarisation : "Souhaitez-vous que votre enfant soit scolarisé : a) dans une école de votre religion; b) dans une école publique sans éducation religieuse ; c) dans une école publique dans laquelle serait possible une éducation religieuse?', en comparant les catholiques français sans ascendance étrangère, les musulmans et les athées dans les deux cas. Les musulmans étaient beaucoup moins en demande d'écoles privées religieuses que les catholiques, avec $3 \%$ contre $12 \%$. Au final, les musulmans français étaient éventuellement en demande d'une adaptation de la politique française dans la vie quotidienne, mais non pas sur les principes. Ce sont des adaptations attendues au niveau local, comme la possibilité de ne pas manger de porc à la cantine. 
L'enquête TeO montre que l'islam n'apparait pas essentiellement sous la forme de "l'islam des mosquées" : la plupart des musulmans de France déclarent avoir une pratique privée de la religion, sans relation avec les mosquées.

\section{H\&M : Le rapport TeO aborde moins la question des carrières politiques, de l'éligibilité et de l'accès, lorsque l'on est algérien ou descendant d'Algériens, à la classe politique française. Est-ce que les recherches que vous avez menées par ailleurs montrent des freins aux carrières poli- tiques des citoyens issus de l'immigration?}

V. T. : Oui, il y a plusieurs freins. Tout d'abord, l'incapacité du système politique français à s'ouvrir au renouvellement. Cela vaut en termes sociaux, de genre et de génération, mais aussi de diversité.

Par ailleurs, la diversité est une question dont la légitimité, en tant que composante sociale, se pose toujours. On s'interroge encore sur la légitimité de la représentation de la diversité, alors que cela n'est plus le cas pour les femmes ni pour les jeunes.

À ces deux points s'en ajoute un troisième. Souvenez-vous de la déclaration de Patrick Ollier, selon lequel s'il n'y a pas de députés de la diversité, c'est parce qu'ils n'en ont pas le niveau. Or il faut savoir que les rares fois où les grands partis, comme le PS et l'UMP, ont fait de la place à des candidats issus de la diversité, c'était souvent dans des circonscriptions qui n'étaient pas gagnables. On a mis en avant, par exemple, une candidate de l'UMP dans les $\mathrm{X}^{\mathrm{c}}$ et XVIII ${ }^{\mathrm{c}}$ arrondissements de Paris, là où la gauche obtient systématiquement des scores au-delà de $60 \%$ : dans un tel contexte, n'importe quel candidat de droite est voué à la défaite. Cela démonte l'argument selon lequel les Français ne seraient pas prêts à accepter des élus issus de la diversité, car il y aurait du racisme et que la promotion de candidats de la diversité ne fonctionnerait donc pas.

Avec Sylvain Brouard, nous avons fait varier les origines du candidat, son genre, son milieu social, ou encore son parti, et avons constaté que l'appréhension du candidat issu de la diversité est plutôt favorable. Au contraire, on le préfère à n'importe quel autre candidat au profil "classique", tout simplement parce qu'il représente une ouverture et un renouvellement politique. Autrement dit, pour résumer, les freins à la représentation de la diversité sont à chercher essentiellement du côté des partis, et beaucoup moins du côté des électeurs, à la différence de ce que l'on pourrait croire.

H\&M : Peut-on comparer la trajectoire des immigrés algériens avec les carrières politiques des immigrés portugais, arrivés en même temps, 


\section{peut-être avec une moins bonne connaissance de la langue et de la culture démocratique françaises?}

V. T. : Le problème auquel nous faisons face est un manque de recul historique suffisant pour aborder ce sujet. Cette recherche comparative fait partie des nouveaux chantiers de la recherche à construire, et devrait être menée de manière collective.

\section{H\&M : Pour finir, je voudrais savoir si, en France, la recherche sur ces thèmes est si riche, ou si, en dehors du CEVIPOF et du CEE, il y a peu d'intérêt pour ces questions.}

V. T. : Ces thèmes suscitent beaucoup d'intérêt, et cela est visible surtout parmi les doctorants, car dans la génération en formation, environ une étude sur trois porte sur la question de la diversité. Ce champ se constitue très vite, et la relève des pionniers comme Vincent Geisser ou Catherine Wihtol de Wenden, par exemple, est en train d'être assurée. Globalement, en termes d'état de la littérature, après les idées de quelques auteurs précurseurs, la fin des années quatre-vingt et le début des années quatre-vingt-dix ont donné lieu à une véritable montée de l'intérêt, qui a connu ensuite un arrêt très dur avec l'affaire d'Hervé Le Bras/Michèle Tribalat et le débat sur le "démon des origines". Depuis 2001, avec le débat autour du voile, la découverte des discriminations, etc., l'enjeu de la diversité est dû notamment à l'effet des études sur le genre. Ces études du genre ont permis de nourrir les études sur la diversité. On voit, par exemple, que des nombreuses chercheures ayant commencé leurs travaux sur la relation homme/femme ont ensuite intégré une réflexion sur la diversité.

Par ailleurs, une autre évolution du champ de la recherche réside dans le passage d'une approche qualitative (par exemple, Nancy Venel ou Camille Hamidi) à une approche quantitative : dans les grandes enquêtes sociologiques, on intègre aujourd'hui des questions sur la diversité, sur les origines. En ce sens, l'enquête TeO va être un bon levier pour l'explication et l'étude de l'immigration en France.

Dans les enquêtes plus traditionnelles auxquelles j'ai participé, on pose la question des origines nationales : "Avez-vous parmi vos parents ou grands-parents quelqu'un qui a eu la nationalité d'un pays étranger ?' C'est une manière d'aborder le sujet de la diversité sans poser de manière directe la question de la couleur de peau.

En même temps, l'un des résultats les plus frappants de l'enquête TeO est la convergence de situations entre les Ultramarins et descendants d'Ultramarins et les immigrés et descendants d'immigrés africains. Les Ultramarins, membres de la communauté française depuis plus longtemps que les Corses, se trouvent confrontés à des préjugés identiques à ceux qui touchent les personnes d'origine africaine. Ce qui montre que la couleur de peau joue beaucoup en France. 